Physica B 165\&166 (1990) 17-18

North-Holland

\title{
THE DEGENERATE-INTERNAL-STATES APPROXIMATION FOR COLD COLLISIONS
}

\section{A.C. MAAN, E. TIESINGA, H.T.C. STOOF, and B.J. VERHAAR}

Department of Physics, Eindhoven University of Technology, Postbus 513, 5600 MB Eindhoven, The Netherlands

The Degenerate-Internal-States approximation as well as its first-order correction are shown to provide a convenient method for calculating elastic and inelastic collision amplitudes for low temperature atomic scattering.

A considerable simplification of (in)elastic collision problems occurs when the energy differences of internal states significantly coupled in a quantum collision are neglected. Usually. this is interpreted classically to correspond to slow internal motion of the collision partners during their interaction time. The first context in which this approximation has been applied was, to the authors' knowledge, the subject of nuclear reactions induced by light projectiles in the fifties (1). In that context it was known as the adiabatic approximation. The same approximation has been applied in connection with heavy ion scattering of nuclei (2) and was then referred to as the sudden approximation. A more recent application occurred under the name

(energy-) sudden approximation in atomic scattering (3), in which case the adjective "energy-" was sometimes used to distinguish it from the closely related "centrifugal-sudden" approximation.

In the past our Eindhoven group has contributed to applications of the above approximation (which we used to refer to as adiabatic approximation) in the field of vibrational and rotational excitation of nuclei in scattering (4). We also showed that it could be applied to hyperfine transitions in atom-atom spin-exchange collisions (5), to inelastic scattering of $\mathrm{H}$ atoms of $f$ the surface of superfluid helium (6) and to scattering of dressed atoms in a microwave trap (7). In the framework of $\mathrm{H}+\mathrm{H}$ spin-exchange collisions in the $H$ maser (5) we have for the first time avoided the name "adiabatic approximation" in view of the different use of this name in connection with the Born-Oppenheimer method in the same paper. In the present contribution we will adhere to the name "Degenerate-Internal-States" (DIS) approximation for the same reason.

To understand the simplifications of scattering problems brought about by all these Identical methods, we write the exact scattering problem in the coupled-channels form

$$
\underline{R}^{\prime \prime}(r)=V(r) \underline{R}(r) \text {. }
$$

where a prime denotes differentiation with respect to the radial distance $r, \underline{R}$ is a vector with components describing the ( $\mathrm{r}$-dependent) probability amplitudes for finding the collision partners in each of the possible internal states and $V$ is a matrix containing internal energles and coupling potentials. The problem of finding solutions to these equations is simplified considerably if there exist $r$-independent Hermitian commutators of $\mathbf{V}$ (constants of the motion): $[C . V]=0$. As is well-known, this gives rise to a partial decoupling of the problem. In our situation we consider commutators which $V$ does not have by itself, but only after subtraction of a constant diagonal matrix $D$. The elements of $D$ are in our case essentially differences of internal energies. The approximation consists of neglecting D.

Commutators of the type C, considered in previous papers. are the collective nuclear coordinates or the projectile angular momentum along the body-fixed symmetry axis in the case of scattering from deformed nuclei, the surface coordinates in the example of inelastic scattering of $H$ atoms off a surface of superfluid helium and the photon number operator for the scattering of dressed atoms.

To illustrate the simplifications brought about by the above-mentioned methods we consider the example of sub-Kelvin collisions of identical electron-spin $1 / 2$ atoms, for example $H, D$, and all alkalis. The DIS approximation then amounts to turning of the hyperfine splitting, but retaining its influence on the electronnucleus spin wave functions. The scattering channels can then be decoupled by transforming to the triplet and singlet channels, leading to the expression

$$
\begin{aligned}
& s_{\{\gamma \delta\},\{\alpha \beta\}}^{\ell, D I S}=\left[k_{\{\gamma \delta\}} k_{\{\alpha \beta\}^{\prime}} \mathbf{k}^{2}\right]^{\ell+1 / 2} \\
& \left(\{\gamma \delta\}\left|\mathrm{e}^{2 \mathrm{i} \delta} \mathrm{S} \mathrm{P}_{\mathrm{S}}+\mathrm{e}^{2 \mathrm{i} \delta} \mathrm{T}_{\mathrm{T}}^{\ell}\right|\{\alpha \beta\}\right),
\end{aligned}
$$


the P operators standing for projection operators on singlet and triplet subspaces, and $k$ standing for the wave number associated with an average total internal energy $\epsilon$. but with the same value of the total energy. The problem is thus reduced to the much simpler problem of calculating the phase shifts for (uncoupled) potential scattering.

In view of the considerable pay-offs associated with the DIS approximation, it is worthwile to extend its range of validity. In 1974 Schulte and Verhaar (4) derived a correction of first order in the non-degeneracy, consisting of a Born type volume integral of the non-degeneracy perturbation, supplemented by a Wronskian surface term. This method was applied to the total threedimensional scattering wave function. More recently, in connection with frequency shifts of the sub-Kelvin hydrogen maser our group showed this approach to be very helpful when applied to individual partial waves. The first-order perturbation due to the hyperfine splitting $\mathrm{H}_{\mathrm{hf}}-\epsilon$ turned out to be

$$
\begin{aligned}
\Delta S_{\{\gamma \delta\},\{\alpha \beta\}}^{\ell}=\left[\mathrm{k}_{\{\gamma \delta\}^{2}} \mathrm{k}_{\{\alpha \beta\}^{-}} \mathbf{k}^{2}\right]^{\ell+1 / 2} \Delta^{\ell} \\
\left\langle\{\gamma \delta\}\left|\left(\mathrm{P}_{\mathrm{T}}-\mathrm{P}_{\mathrm{S}}\right)\left(\mathrm{H}_{\mathrm{hf}}-\epsilon\right)\left(\mathrm{P}_{\mathrm{T}}-\mathrm{P}_{\mathrm{S}}\right)\right|\{\alpha \beta\}\right\rangle
\end{aligned}
$$

For an application of these methods to spinexchange collisions in the cold deuterium maser we refer to another contribution to this conference (8).

\section{REFERENCES}

(1) S.I. Drozdov, Zh. Eksp. Teor. Fiz. 28 (1955) 734,736 [Sov. Phys. JETP 1 (1955) 588, 591]; D.M. Chase, Phys. Rev. 104 (1956) 838; C.A. Levinson, Nuclear Spectroscopy B (Academic Press. New York, 1960) p. 677.

(2) K. Alder and A. Winther, Mat. Fys. Medd. 32 (1960) No. 8.

(3) P. McGuire and D.J. Kouri, J. Chem. Phys. 60 (1974) 2488.

(4) A.M. Schulte and B.J. Verhaar, Nucl. Phys. A232 (1974) 215; B.J. Verhaar and A.M. Schulte, Phys. Lett. 67B (1977) 381.

(5) B.J. Verhaar. J.M.V.A. Koelman. H.T.C. Stoof, O.J. Luiten, and S.B. Crampton, Phys. Rev. A 35 (1987) 3825; J.M.V.A. Koelman, S.B. Crampton, H.T.C. Stoof. O.J. Luiten, and B.J. Verhaar, Phys. Rev. A 38 (1988) 3535.

(6) E. Tiesinga, H.T.C. Stoof, and B.J. Verhaar, Phys. Rev. B (to appear).

(7) C.C. Agosta, I.F. Silvera, H.T.C. Stoof, and B.J. Verhaar. Phys. Rev. Lett. 62 (1989) 2361.

(8) E. Tlesinga, H.T.C. Stoof, B.J. Verhaar, and S.B. Crampton, Spin-exchange frequency shift of the cryogenic deuterium maser, this volume. 